\title{
Time-resolved spectroscopy of the planet-hosting sdB pulsator V391 Pegasi
}

\author{
S. Schuh, ${ }^{1}$ R. Kruspe, ${ }^{1}$ R. Lutz, ${ }^{1}$ and R. Silvotti ${ }^{2}$ \\ ${ }^{1}$ Institut für Astrophysik, Universität Göttingen, Friedrich-Hund-Platz 1, 37077 Göttingen, Germany \\ 2 INAF - Osservatorio Astronomico di Capodimonte, via Moiariello 16, 80131 Napoli, Italy
}

\begin{abstract}
The subdwarf B (sdB) star V391 Peg oscillates in short-period p modes and long-period $\mathrm{g}$ modes, making it one of the three known hybrids among sdBs. As a by-product of the effort to measure secular period changes in the $\mathrm{p}$ modes due to evolutionary effects on a time scale of almost a decade, the $\mathrm{O}-\mathrm{C}$ diagram has revealed an additional sinusoidal component attributed to a periodic shift in the light travel time caused by a planetary-mass companion around the sdB star in a $3.2 \mathrm{yr}$ orbit. In order to derive the mass of the companion object, it is necessary to determine the orbital inclination. One promising possibility to do this is to use the stellar inclination as a primer for the orbital orientation. The stellar inclination can refer to the rotational or the pulsational axis, which are assumed to be aligned, and can in turn then be derived by combining measurements of $v_{\text {rot }}$ and $v_{\text {rot }} \sin i$.

The former is in principle accessible through rotational splitting in the photometric frequency spectrum (which has however not been found for V391 Peg yet), while the projected rotational velocity can be measured from the rotational broadening of spectral lines. The latter must be deconvolved from the additional pulsational broadening caused by the surface radial velocity variation in high $\mathrm{S} / \mathrm{N}$ phase averaged spectra. This work gives limits on pulsational radial velocities from a series of phase resolved spectra.

Phase averaged and phase resolved high resolution echelle spectra were obtained in May and September 2007 with the 9m-class Hobby-Eberly Telescope (HET), and one phase averaged spectrum in May 2008 with the $10 \mathrm{~m}$-Keck 1 telescope ${ }^{1}$.
\end{abstract}

Individual Objects: V391 Pegasi

\section{The hybrid pulsating sdB star V391 Pegasi and its planetary companion}

Subdwarf B stars (sdBs) are subluminous, evolved stars on the extreme horizontal branch (EHB). They have a He burning core but, due to previous significant mass loss, no $\mathrm{H}$ shell burning in their thin hydrogen shells. Their masses cluster around $0.5 \mathrm{M}_{\odot}$. Only a small fraction of the sdBs show pulsational variations, with non-pulsators also populating the region in the HRD where the pulsators are found. There are $p$ (pressure) mode and $g$ (gravity) mode types of pulsation. Three objects that show both mode types are referred to as hybrid pulsators and among them is V391 Peg (HS 2201+2610) which has five p modes (Østensen et al. 2001; Silvotti et al. 2002) and one g mode (Lutz et al. 2008, 2009). Silvotti

\footnotetext{
${ }^{1}$ Data obtained with the Hobby-Eberly Telescope (joint project of $U$ of Texas, Pennsylvania State $U$, Stanford U, $U$ München, U Göttingen) and the W.M. Keck Observatory (operated by CalTech, U of California, NASA), made possible by the generous financial support of the W.M. Keck Foundation.
} 


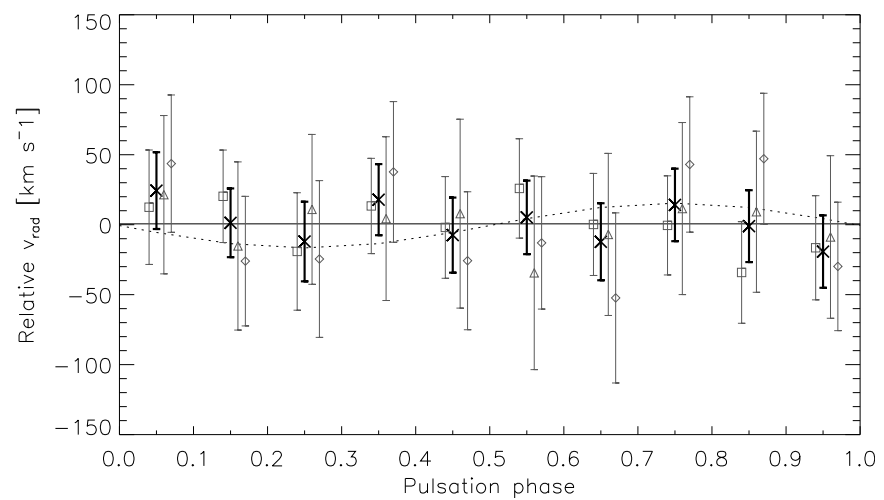

Figure 1: Radial velocities derived for the $\mathrm{H} \beta$ (squares), $\mathrm{H} \gamma$ and $\mathrm{H} \delta$ (triangles and diamonds) lines from cross correlation with the template (with error bars, and plotted at a small phase offset for clarity); and weighted mean of the results (crosses and thick error bars) together with a constant and a sinusoidal curve.

et al. (2007) detected parabolic and sinusoidal variations in the observed-calculated (O-C) diagram constructed for the two main pulsation frequencies at $349.5 \mathrm{~s}$ and $354.2 \mathrm{~s}$ over the observing period of seven years. The sinusoidal component is attributed to the presence of a very low-mass companion (V391 Peg b, $m \sin i=3.2 \pm 0.7 \mathrm{M}_{\text {Jup }}$ ). The determination of the true mass of this 'asteroseismic planet' requires a constraint on the orbital inclination which can presumably be determined via the stellar rotational inclination.

\section{Limits on the pulsational radial velocities from phase resolved echelle spectra}

Echelle spectra of V391 Peg were taken during May and September 2007 with the HRS $(R=15000)$ of the HET at the McDonald Observatory, and with HIRES $(R=31000)$ at the Keck 1 telescope atop Mauna Kea in May 2008. Data reduction was done with ESOMidas using standard procedures. Individual echelle orders were merged and the final spectra were carefully normalized and finally summed (Kruspe 2009, diploma thesis, in prep.). This results in a set of individual spectra $(S / N \approx 3)$, in particular two September 2007 high time resolution series, and summed spectra for May and September 2007.

In our attempt to "clean" the relevant rotational broadening from pulsational effects, the spectra in September obtained in time resolved mode were combined to a series of ten phase resolved averaged spectra $(S / N \approx 9)$ for the main pulsation period of $349.5 \mathrm{~s}$ (similar to Tillich et al. 2007).

The cross-correlation of this series of averaged spectra with a pure hydrogen NLTE model spectrum at $T_{\text {eff }}=30000 \mathrm{~K}$ and $\log \left(\mathrm{g} / \mathrm{cm} \mathrm{s}^{-2}\right)=5.5$ as a template yields pulsational radial velocity measurements as shown in Fig. 1 for the different Balmer lines. The maximum amplitude of a sinusoidal curve (fixed at the expected period) that could be accommodated in comparison to the weighted means of the Balmer lines reveals that any pulsational radial velocity amplitude is smaller than the accuracy of our measurements and confirms the upper limit of $16 \mathrm{~km} \mathrm{~s}^{-1}$ given by Kruspe et al. (2008).

The resolution of the model template matches the spectral resolution of the (pulsationaveraged) Keck spectrum. A comparison of the $\mathrm{H} \alpha$ NLTE line core shape yields an even more stringent upper limit for the combined broadening effect of pulsation and rotation of at most $9 \mathrm{~km} \mathrm{~s}^{-1}$. This means that much better data in terms of spectral resolution and signal to noise will be necessary to measure $v_{\text {puls }}$ and $v_{\text {rot }} \sin i$. 
Acknowledgments. The authors thank A. Reiners and G. Basri for providing the Keck spectrum, H. Edelmann for assistance in obtaining the HET spectra, and U. Heber and T. Rauch for providing grids of model spectra. This work has benefited from the help, advice and software by I. Traulsen. We also thank the conference sponsors and in particular HELAS (European Helio- and Asteroseismology Network, an European initiative funded by the European Commission since April 1st, 2006, as a "Co-ordination Action" under its Sixth Framework Programme FP6) and the Astronomische Gesellschaft for financially supporting the poster presentation at JENAM 2008 Minisymposium $N^{\circ} 4$ through travel grants to S.S. and R.L.

\section{References}

Kruspe, R., Schuh, S., Silvotti, R., \& Traulsen, I. 2008, CoAst, 157, 325

Lutz, R., Schuh, S., Silvotti, R., et al. 2008, ASP Conf. Ser., 392, 339

Lutz, R., Schuh, S., Silvotti, R., et al. 2009, A\&A, 496, 469

Østensen, R., Solheim, J.-E., Heber, U., et al. 2001, A\&A, 368, 175

Silvotti, R., Janulis, R., Schuh, S., et al. 2002, A\&A, 389, 180

Silvotti, R., Schuh, S., Janulis, R., et al. 2007, Nature, 449, 189

Tillich, A., Heber, U., O'Toole, S. J., et al. 2007, A\&A, 473, 219

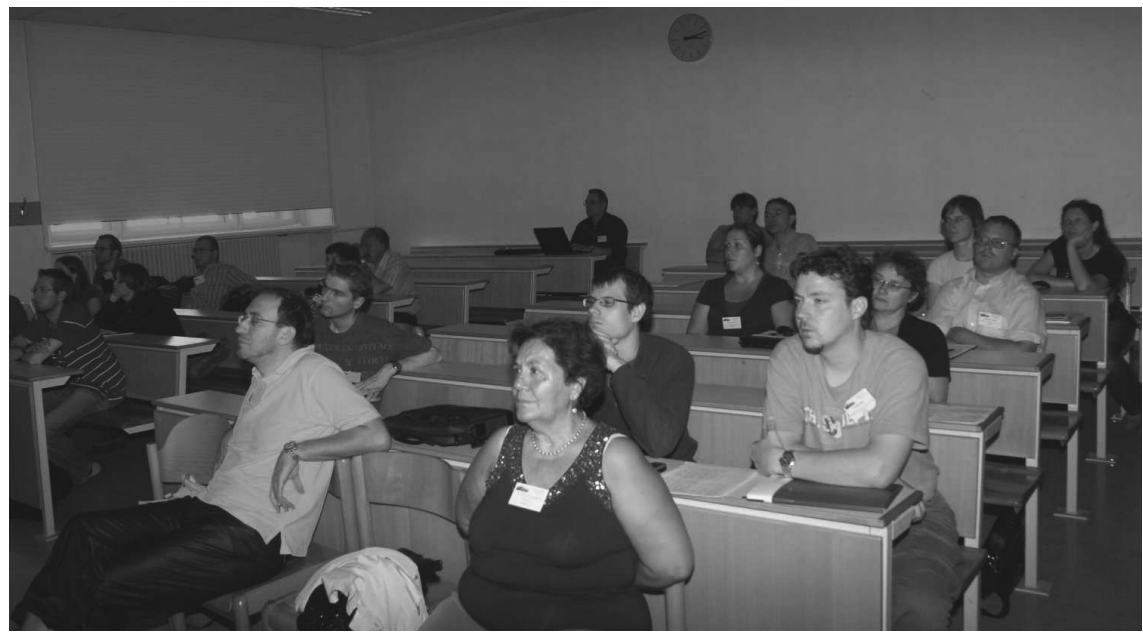

